\title{
FORMAS DE FÓSFORO EM SOLOS SOB INFLUÊNCIA DA CALAGEM E ADUBAÇÃo ORGÂNICA ${ }^{(1)}$
}

\author{
Renato Ferreira de Souza ${ }^{(2)}$, Valdemar Faquin ${ }^{(3)}$, Alex Teixeira de \\ Andrade $^{(4)} \&$ Paulo Rogério Ferreira Torres ${ }^{(5)}$
}

\begin{abstract}
RESUMO
A distribuição das formas de $P$ nos diferentes compartimentos do solo (lábil, pouco lábil e não-lábil) é amplamente influenciada por processos geoquímicos e biológicos e, também, por ações antrópicas. Visando avaliar a influência da calagem e do esterco bovino sobre a distribuição do $P$ nos diferentes compartimentos do solo, foram realizados em casa de vegetação quatro experimentos no delineamento inteiramente ao acaso, em esquema fatorial $4 \times 5$, com quatro repetições. Os tratamentos foram constituídos por quatro doses de calcário $(0 ; 0,5 ; 1 ;$ e 2 vezes a dose recomendada para atingir $V=60 \%)$ e cinco doses de esterco bovino $(0 ; 2,5 ; 5,0$; 7,5; e $10 \%$ do volume total de solo), aplicadas em amostras de $4 \mathrm{dm}^{3} \mathrm{de}$ Neossolo Quartzarênico órtico (RQo), Latossolo Vermelho-Amarelo distrófico textura média (LVAd-1), Latossolo Vermelho-Amarelo distrófico textura argilosa (LVAd-2) e Latossolo Vermelho distrófico textura muito argilosa (LVd), sendo cada solo um experimento. Foram avaliados os teores de $P$ Mehlich-1, $P$ lábil (P resina + Pi e Po$\mathrm{NaHCO}_{3}$ ), P pouco lábil (Pi e Po-NaOH) e P não-lábil (P-HCl + P-residual). De maneira geral, a adição de calcário e esterco bovino incrementou os teores de $P$ Mehlich-1, principalmente nos solos menos oxídicos e com textura mais arenosa. Houve aumento em todas as formas de $P$ nos solos, sendo a contribuição das formas pouco lábeis (Po ligado a compostos húmicos e Pi ligado a Fe e Al) bastante substancial, predominando, porém, as formas não-lábeis de $P$.
\end{abstract}

Termos de indexação: esterco bovino, calcário, disponibilidade de fósforo, P Mehlich-1.

\footnotetext{
${ }^{(1)}$ Parte da Tese de Doutorado apresentada pelo primeiro autor à Universidade Federal de Lavras - UFLA. Projeto financiado pela FAPEMIG e CNPq. Recebido para publicação em novembro de 2005 e aprovado em julho de 2007.

${ }^{(2)}$ Doutor em Solos e Nutrição de Plantas, Departamento de Ciência do Solo, Universidade Federal de Lavras - UFLA. Rua José Claudino 268. CEP 37200-000 Lavras (MG). Assistente de Pesquisa PRODOC-CAPES. E-mail: souzarf.ufla@gmail.com

(3) Professor Titular do Departamento de Ciência do Solo, UFLA. Bolsista CNPq. E-mail: vafaquin@ufla.br

${ }^{(4)}$ Doutor em Solos e Nutrição de Plantas, EMATER/MG, DCS/UFLA.

${ }^{(5)}$ Graduando em Agronomia, UFLA. E-mail: paulorogerioftorres@gmail.com
} 


\title{
SUMMARY: PHOSPHORUS FORMS IN SOILS UNDER INFLUENCE OF LIMING AND ORGANIC FERTILIZATION
}

\begin{abstract}
The distribution of phosphorus forms in the different soil compartments (labile, little labile and non-labile) is very influenced by geochemical and biological processes and by human activities. To evaluate the effect of liming and cattle manure on soil phosphorus forms, four experiments were carried out in an entirely randomized design, in a $4 \mathrm{x} 5$ factorial layout with four repetions, in a greenhouse. The treatments consisted of four liming levels (0; $0.5 ; 1$ and 2 times the recommended dose to reach $V=60 \%)$ and five doses of cattle manure $\left(0 ; 2.5 ; 5.0 ; 7.5\right.$ and $10 \%$ of the total soil volume), applied on samples of four $\mathrm{dm}^{3}$ of four soil types: sandy,orthic Quartzarenic Neosol, loamy clay dystrophic Red-Yellow Latosol, sandy clayey dystrophic Red-Yellow Latosol and a clayey dystrophic Red Latosol. Each soil represented an experiment. The concentrations of $P$ (Mehlich-1) and the distribution of the labile (P-resin + Pi and $\mathrm{Po}-\mathrm{NaHCO}_{3}$ ), little labile ( $\mathrm{Pi}$ and $\mathrm{Po}-\mathrm{NaOH}$ ) and non-labile $(P-H C l+P$-residual) forms of phosphorus in the soil were evaluated. In general, the increment in the doses of cattle manure and lime promoted a significant increase in $P$ (Mehlich-1) concentrations in the soil, mainly in the less oxidic, sandier. All P forms in the soils increased, but contribution of the little labile forms (Po linked to humic compounds and $\mathrm{Pi}$ bound to $\mathrm{Fe}$ and $\mathrm{Al}$ ) was rather substantial, although the non-labile P form prevailed in these soils.
\end{abstract}

Index terms: cattle manure, lime, Mehlich-1 P, phosphorus availability.

\section{INTRODUÇÃO}

O fósforo encontra-se nos solos sob as formas orgânicas (Po) e inorgânicas (Pi), que se diferenciam entre si pelo grau de estabilidade ou solubilidade e, portanto, com diferentes disponibilidades à absorção vegetal. O Pi pode se encontrar em solução (P-solução), precipitado com $\mathrm{Al}$, $\mathrm{Fe}$ e Ca ou adsorvido aos óxidos de $\mathrm{Fe}$ e $\mathrm{Al}$ da fração argila, ambos em equilíbrio com a solução e compondo a fração lábil de P no solo (P lábil). A fração não-lábil é composta pelo $\mathrm{P}$ sob a forma de compostos de baixa solubilidade (P mineralogicamente estável) ou adsorvido às partículas do solo, não estando, estas formas, em equilíbrio imediato com o $\mathrm{P}$ em solução (Novais \& Smyth, 1999). Em ecossistemas naturais, os processos geoquímicos são responsáveis pela dinâmica do $\mathrm{P}$ em longo prazo. Contudo, pelo fato de a maior parte do $\mathrm{P}$ disponível às plantas ter como fonte a matéria orgânica, os processos biológicos influenciam bastante a distribuição das formas de $\mathrm{P}$ no solo (Walbridge, 1991). Portanto, os processos biológicos regulam a dinâmica e distribuição de formas lábeis de $\mathrm{P}$ no solo, e a reciclagem da forma orgânica é um importante fator na disponibilização desse nutriente às plantas (Adeptu \& Corey, 1976).

O manejo do solo, as remoções de $\mathrm{P}$ pelas culturas, as aplicações de fertilizantes fosfatados, a calagem e adição de matéria orgânica alteram bastante a dinâmica das transformações do P no solo (Tiessen et al., 1992). Em estudos realizados em cafeeiros cultivados em Latossolo Roxo distrófico foi observado que as principais transformações de $\mathrm{P}$ foram decorrentes das alterações nos valores de $\mathrm{pH}$ e nos teores de matéria orgânica desse solo (Pavan \& Chaves, 1996). Estudos têm demonstrado que a natureza e dinâmica da matéria orgânica e a ciclagem de $\mathrm{P}$ apresentam comportamentos diferentes sob diferentes sistemas de manejo do solo. Portanto, práticas de cultivo que alteram o comportamento da matéria orgânica exercem grande impacto sobre a distribuição das formas de P no solo (Turrión et al., 2000). Vários trabalhos que visam entender a dinâmica do $\mathrm{P}$ têm demonstrado a influência de diferentes práticas - como a calagem, a adição de fontes ou doses de matéria orgânica e os sistemas de cultivo - sobre as transformações das formas de P no solo (Hedley et al., 1982; Iyamuremye et al., 1996; Tokura et al., 2002).

Devido ao comportamento do $\mathrm{P}$ e para melhor entendimento de sua dinâmica no solo, têm sido realizados diversos trabalhos de fracionamento seqüencial empregando soluções químicas com diferentes capacidades de extração de P (Silva \& Raij, 1999). O método de fracionamento seqüencial, proposto por Hedley et al. (1982), tem sido amplamente empregado no estudo das transformações do P em razão das diferentes práticas de manejo do solo, com o objetivo de incrementar os conhecimentos sobre sua dinâmica e predição de sua biodisponibilidade e riscos de perdas (Hedley et al., 1982; Iyamuremye et al., 1996; Tokura et al., 2002).

Objetivou-se com este estudo avaliar o efeito de doses de calcário e de esterco bovino sobre as formas lábeis, pouco lábeis e não-lábeis de $\mathrm{P}$ em quatro solos bem drenados, com diferentes textura, mineralogia e teor de matéria orgânica. 


\section{MATERIAL E MÉTODOS}

Quatro experimentos foram realizados no Departamento de Ciência do Solo da Universidade Federal de Lavras, com amostras de quatro solos bem drenados, predominantes no Estado de Minas Gerais, e com ampla variação nos conteúdos de argila, na mineralogia e nos teores de matéria orgânica (MO), constituindo cada solo um experimento específico. Para abranger ampla variação nas características físicas, químicas e mineralógicas, foram utilizados os solos classificados no Sistema Brasileiro de Classificação de Solos (Embrapa, 1999) como: Neossolo Quartzarênico órtico fase cerrado (RQo), Latossolo Vermelho-Amarelo distrófico textura média fase floresta tropical subperenifólia (LVAd-1), Latossolo Vermelho-Amarelo distrófico textura argilosa fase floresta tropical subperenifólia (LVAd-2) e Latossolo Vermelho distrófico textura muito argilosa fase floresta tropical subperenifólia (LVd).

As amostras dos solos foram coletadas na profundidade de $0-20 \mathrm{~cm}$, sob vegetação natural, após retirar a vegetação e os restos orgânicos presentes na superfície. Após coleta, as amostras foram caracterizadas física, química e mineralogicamente (Quadro 1). A granulometria dos solos foi determinada pelo méto- do da pipeta (Day, 1965), empregando-se $\mathrm{NaOH}$ $0,1 \mathrm{~mol} \mathrm{~L}^{-1}$ como dispersante químico e agitação rápida, sendo a fração areia $(2-0,053 \mathrm{~mm})$ separada por meio de tamisagem. $\mathrm{O} \mathrm{pH}$ em água, $\mathrm{Ca}^{2+}, \mathrm{Mg}^{2+}, \mathrm{Al}^{3+}$, $\mathrm{P}, \mathrm{K}, \mathrm{Cu}, \mathrm{Fe}, \mathrm{Mn}$ e $\mathrm{Zn}$ foram determinados conforme Embrapa (1997), sendo o $\mathrm{Ca}^{2+}, \mathrm{Mg}^{2+} \mathrm{e} \mathrm{Al}^{3+}$ extraídos com $\mathrm{KCl} 1 \mathrm{~mol} \mathrm{~L}^{-1}$, e $\mathrm{P}, \mathrm{K}$ e os micronutrientes catiônicos, pelo $\mathrm{HCl} 0,05 \mathrm{~mol} \mathrm{~L}^{-1}+\mathrm{H}_{2} \mathrm{SO}_{4}$ $0,0125 \mathrm{~mol} \mathrm{~L}^{-1}$ (Mehlich-1). Também foram determinados a acidez potencial $(\mathrm{H}+\mathrm{Al})$ e o $\mathrm{C}$ orgânico, conforme Raij et al. (1987). O P remanescente foi determinado conforme Alvarez V. et al. (2000), e a capacidade máxima de adsorção de $\mathrm{P}$, segundo Olsen \& Watanabe (1957); os valores de P adsorvido foram ajustados à isoterma de Langmuir, para estimativa da CMAP. Na fração argila foram quantificadas caulinita $(\mathrm{Ct})$ e gibbsita, $(\mathrm{Gb})$ por análise termodiferencial (ATD), sendo as amostras da mesma fração submetidas à difração de raios $\mathrm{X}$ (método do pó) (Klug \& Alexander, 1974); óxidos de Fe livre totais $\left(\mathrm{Fe}_{\mathrm{d}}\right)$, usando o ditionito-citrato-bicarbonato de sódio (DCB) (Mehra \& Jackson, 1960); e óxidos de Fe menos cristalinos $\left(\mathrm{Fe}_{0}\right)$, extraídos segundo método de Schwertmann (1964), usando-se oxalato ácido de amônio; em ambos os casos a dosagem do $\mathrm{Fe}$ foi realizada por espectrofotometria de absorção atômica

Quadro 1. Principais atributos químicos, físicos e mineralógicos das amostras dos solos, coletadas sob condições naturais, na camada de 0 a $20 \mathrm{~cm}$

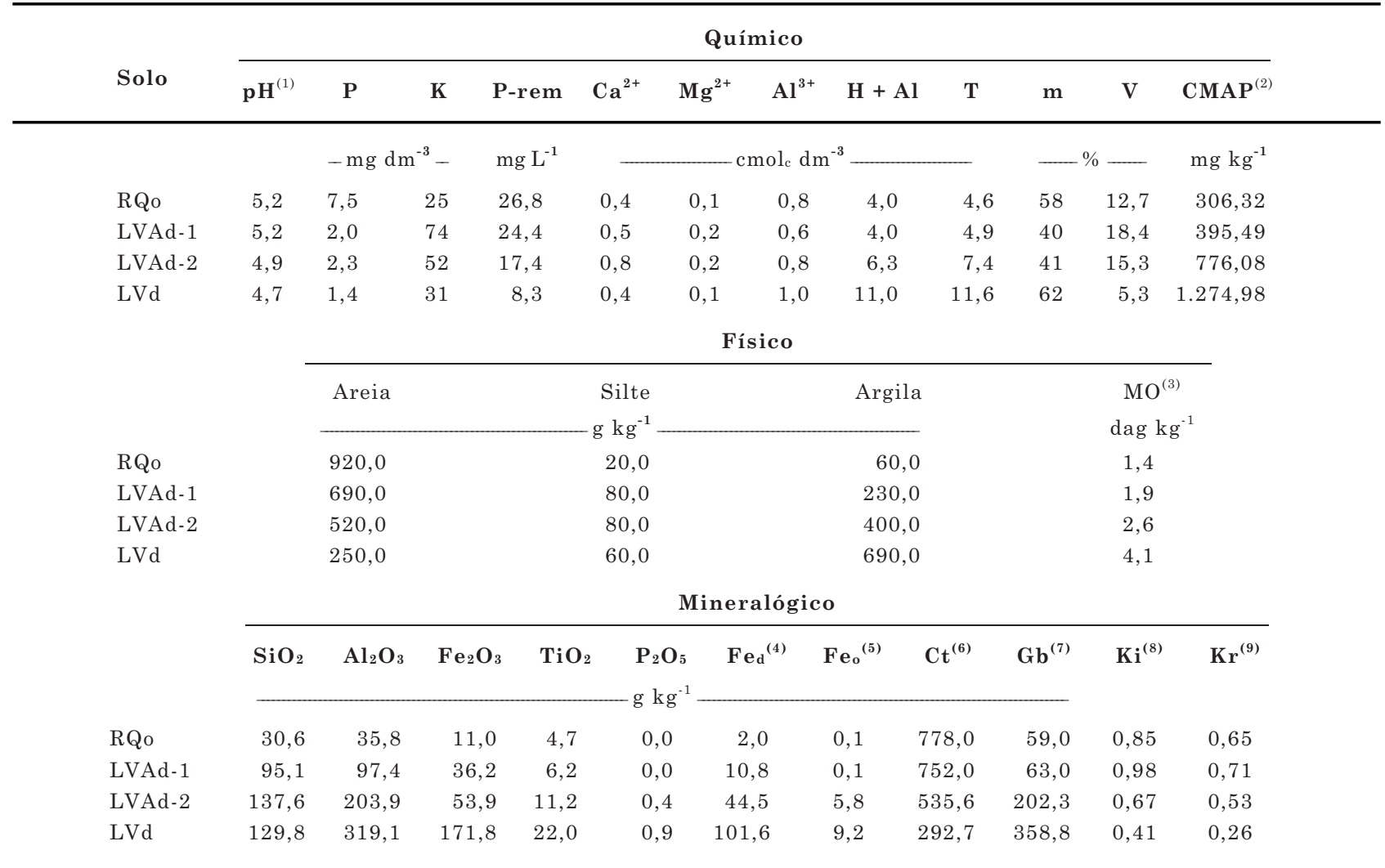

${ }^{(1)} \mathrm{pH}$ em água. ${ }^{(2)}$ capacidade máxima de adsorção de $\mathrm{P} .{ }^{(3)} \mathrm{MO}=\% \mathrm{C}$ x $1,723 .{ }^{(4)}$ óxidos de Fe livre totais. ${ }^{(5)}$ óxidos de $\mathrm{Fe}$ menos cristalinos. ${ }^{(6)}$ caulinita. ${ }^{(7)}$ gibbsita. ${ }^{(8)} \mathrm{Ki}=\mathrm{SiO}_{2} / \mathrm{Al}_{2} \mathrm{O}_{3} \cdot{ }^{(9)} \mathrm{Kr}=\mathrm{SiO}_{2} / \mathrm{Al}_{2} \mathrm{O}_{3}+\mathrm{Fe}_{2} \mathrm{O}_{3}$. 
(Krishna Murti et al., 1966). Os óxidos foram determinados por ataque sulfúrico $\left(\mathrm{SiO}_{2}, \mathrm{Al}_{2} \mathrm{O}_{3}, \mathrm{Fe}_{2} \mathrm{O}_{3}, \mathrm{TiO}_{2}\right.$ e $\mathrm{P}_{2} \mathrm{O}_{5}$ ), segundo Embrapa (1997), e determinadas as relações moleculares $\mathrm{Ki}=\mathrm{SiO}_{2} / \mathrm{Al}_{2} \mathrm{O}_{3}$ e $\mathrm{Kr}=\mathrm{SiO}_{2} /$ $\mathrm{Al}_{2} \mathrm{O}_{3}+\mathrm{Fe}_{2} \mathrm{O}_{3}$.

Os experimentos foram realizados em casa de vegetação, utilizando-se o delineamento experimental inteiramente casualizado (DIC), em esquema fatorial $4 \times 5$, com quatro repetições, sendo cada unidade experimental constituída por um vaso com capacidade de $4 \mathrm{dm}^{3}$ de solo. Os tratamentos foram constituídos de quatro doses de calcário, equivalentes a 0,$0 ; 0,5$; 1,$0 ;$ e 2,0 vezes as doses recomendadas para elevar a saturação por bases em cada solo a $60 \%$, e cinco doses de esterco bovino decomposto $(0,100,200,300 \mathrm{e}$ $400 \mathrm{~cm}^{3} /$ vaso), correspondendo a 0,$0 ; 2,5 ; 5,0 ; 7,5$; e $10 \%$ do volume de solo de cada parcela experimental.

Para sua incorporação aos solos, o esterco bovino foi seco a $60{ }^{\circ} \mathrm{C}$ e moído em moinho com malha de $1 \mathrm{~mm}$, apresentando densidade de $0,462 \mathrm{~kg} \mathrm{dm}^{-3}$; a análise química do esterco apresentou os seguintes teores de nutrientes: 18,$0 ; 3,4 ; 14,2 ; 12,1 ; 6,0$; e $2,6 \mathrm{~g} \mathrm{~kg}^{-1}$ de N, P, K, Ca, Mg e S e 21,9; 1.112,8; 449,4; e 78,5 $\mathrm{mg} \mathrm{kg}^{-1}$ de $\mathrm{Cu}, \mathrm{Fe}, \mathrm{Mn}$ e $\mathrm{Zn}$, respectivamente, e $52 \mathrm{dag} \mathrm{kg}^{-1}$ de matéria orgânica.
Utilizou-se calcário dolomítico calcinado e micropulverizado, com $35 \%$ de $\mathrm{CaO}, 14 \%$ de $\mathrm{MgO}$ e PRNT de $97 \%$.

Após o período de 30 dias de incubação do solo com esterco bovino e calagem, cada unidade experimental recebeu as doses de 400, 320, 250 e $200 \mathrm{mg} \mathrm{dm}^{-3} \mathrm{de} \mathrm{P}$ na forma de $\mathrm{Ca}\left(\mathrm{H}_{2} \mathrm{PO}_{4}\right)_{2} \cdot \mathrm{H}_{2} \mathrm{O}$, de acordo com Alvarez V. \& Fonseca (1990), em função dos teores de P-rem apresentados pelos solos LVd, LVAd-2, LVAd-1 e RQo, respectivamente, e uma adubação básica com macro e micronutrientes, utilizando-se como fontes reagentes p.a. Após mais 30 dias de incubação, com umidade próxima à capacidade de campo, subamostras foram coletadas para as determinações analíticas. O P Mehlich-1 foi extraído pelo $\mathrm{HCl} 0,05 \mathrm{~mol} \mathrm{~L}^{-1}+$ $\mathrm{H}_{2} \mathrm{SO}_{4}$ 0,0125 mol L-1, conforme Embrapa (1997), e o fracionamento seqüencial das formas de $\mathrm{P}$ nos solos foi realizado de acordo com o método modificado de Hedley et al. (1982) (Figura 1).

Por esse procedimento de fracionamento seqüencial, as formas lábeis de $\mathrm{P}$ foram extraídas pela resina de troca aniônica ( $\mathrm{P}$ resina) saturada com bicarbonato; o P inorgânico (Pi-NaHCO ${ }_{3}$ ) e orgânico $\left(\mathrm{Po}-\mathrm{NaHCO}_{3}\right)$ adsorvidos nas superfícies dos colóides foram extraídos com $\mathrm{NaHCO}_{3}\left(0,5 \mathrm{~mol} \mathrm{~L}^{-1}\right.$, a pH 8,5). As formas de $\mathrm{P}$

\begin{tabular}{|c|c|c|}
\hline \begin{tabular}{|c|} 
PROCEDIMENTO \\
\end{tabular} & FORMA I & \multirow{3}{*}{ 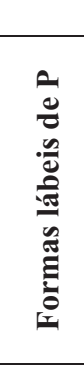 } \\
\hline $\begin{array}{l}1,5 \mathrm{~g} \text { de solo }+50 \mathrm{~mL} \text { água }+ \text { resina. Agitar } 16 \mathrm{~h} . \\
\text { Resina }+\mathrm{HCl} 0,5 \mathrm{~mol} \mathrm{~L}^{-1} . \text { Agitar. }\end{array}$ & P-resina & \\
\hline $\begin{array}{l}\text { Após centrifugar e descartar o sobrenadante, } \\
\text { adicionar } 30 \mathrm{~mL} \mathrm{NaHCO} 0,5 \mathrm{~mol} \mathrm{~L}^{-1} \text {, agitar } 16 \mathrm{~h} \text { e centrifugar. } \\
\text { Numa alíquota: acidificar com } \mathrm{H}_{2} \mathrm{SO}_{4} \text { e resfriar. } \\
\text { Noutra: autoclavagem com persulfato de amônio }+\mathrm{H}_{2} \mathrm{SO}_{4} \text {. } \\
\text { Pt-NaHCO }_{3}-\mathrm{Pi}-\mathrm{NaHCO}_{3}\end{array}$ & $\begin{array}{l}\mathrm{Pi}-\mathrm{NaHCO}_{3} \\
\mathrm{Pt}-\mathrm{NaHCO}_{3} \\
\mathrm{Po}-\mathrm{NaHCO}_{3}\end{array}$ & \\
\hline $\begin{array}{l}\text { Após centrifugar e descartar o sobrenadante, } \\
\text { adicionar } 30 \mathrm{~mL} \mathrm{NaOH} 0,1 \mathrm{~mol} \mathrm{~L}{ }^{-1} \text {, agitar } 16 \mathrm{~h} \text { e centrifugar. } \\
\text { Numa alíquota: acidificar com } \mathrm{H}_{2} \mathrm{SO}_{4} \text { e resfriar. } \\
\text { Noutra: autoclavagem com persulfato de amônio }+\mathrm{H}_{2} \mathrm{SO}_{4} \text {; } \\
\text { Pt-NaOH - Pi-NaOH }\end{array}$ & $\begin{array}{l}\mathrm{Pi}-\mathrm{NaOH} \\
\mathrm{Pt}-\mathrm{NaOH} \\
\mathrm{Po}-\mathrm{NaOH} \\
\end{array}$ & \multirow{2}{*}{ 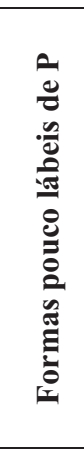 } \\
\hline $\begin{array}{l}\text { Após centrifugar e descartar o sobrenadante, } \\
\text { adicionar } 30 \mathrm{~mL} \mathrm{NaOH} 0,5 \mathrm{~mol} \mathrm{~L}^{-1} \text {, agitar } 16 \mathrm{~h} \text { e centrifugar. } \\
\text { Numa alíquota: acidificar com } \mathrm{H}_{2} \mathrm{SO}_{4} \text { e resfriar. } \\
\text { Noutra: autoclavagem com persulfato de amônio }+\mathrm{H}_{2} \mathrm{SO}_{4} \text {. } \\
\mathrm{Pt}-\mathrm{NaOH}-\mathrm{Pi}-\mathrm{NaOH}\end{array}$ & $\begin{array}{l}\mathrm{Pi}-\mathrm{NaOH} \\
\mathrm{Pt}-\mathrm{NaOH} \\
\mathrm{Po}-\mathrm{NaOH} \\
\end{array}$ & \\
\hline $\begin{array}{l}\text { Após centrifugar e descartar o sobrenadante, } \\
\text { adicionar } 30 \mathrm{~mL} \mathrm{HCl} 1 \mathrm{~mol} \mathrm{~L}^{-1} \text {, agitar } 16 \mathrm{~h} \text { e centrifugar. }\end{array}$ & $\mathrm{P}-\mathrm{HCl}$ & \multirow{2}{*}{ 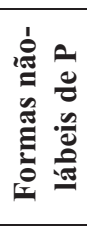 } \\
\hline $\begin{array}{l}0,5 \text { g resíduo do solo após a secagem; } \\
\text { Digestão a } 360{ }^{\circ} \mathrm{C} \text { com } \mathrm{H}_{2} \mathrm{SO}_{4} \text { e } \mathrm{H}_{2} \mathrm{O}_{2} .\end{array}$ & P-residual & \\
\hline
\end{tabular}

Figura 1. Procedimento simplificado de fracionamento seqüencial de fósforo (modificado de Hedley et al., 1982). 
pouco lábil, correspondentes às formas inorgânicas ligadas a $\mathrm{Fe}$ e $\mathrm{Al}(\mathrm{Pi}-\mathrm{NaOH})$ e orgânicas associadas com compostos húmicos (Po-NaOH), foram extraídas com $\mathrm{NaOH}\left(0,1\right.$ e $\left.0,5 \mathrm{~mol} \mathrm{~L}^{-1}\right)$. As formas de $\mathrm{P}$ relativamente insolúveis (P não-lábil), associadas com cálcio, foram extraídas com $\mathrm{HCl}\left(1,0 \mathrm{~mol} \mathrm{~L}^{-1}\right)(\mathrm{Pi}-\mathrm{HCl})$, e o $\mathrm{P}$ residual, contendo as formas mais estáveis de Po e insolúveis de $\mathrm{Pi}$, foi extraído por digestão com $\mathrm{H}_{2} \mathrm{SO}_{4}$ concentrado e $\mathrm{H}_{2} \mathrm{O}_{2}$ (P-residual). Em todos os extratos de solo, o $\mathrm{P}$ foi determinado por colorimetria, segundo Murphy \& Riley (1962).

Os valores de P Mehlich-1 e teores de matéria orgânica após aplicação dos tratamentos foram submetidos ao ajuste de modelos de regressão múltipla (superfícies de resposta), considerando as doses de calcário e de resíduo aplicadas (Alvarez V., 1994). Os modelos ajustados foram selecionados conforme $o$ procedimento stepwise, isto é, considerando um modelo geral completo, selecionou-se um modelo menor (submodelo), no qual todos os coeficientes foram significativos (Khuri \& Cornell, 1996). A adoção do sistema linear formado pelas derivadas parciais das equações da superfície de resposta igualada a zero forneceu os valores críticos, que corresponderam aos valores máximos, de acordo com o comportamento específico da variável P Mehlich-1. Foi utilizado o programa de análises estatísticas $R$ ( $R$ Development Core Team, 2004). As formas de P lábeis, pouco lábeis e não-lábeis foram submetidas à análise de variância e ao estudo de regressão simples, ajustando-se modelos em função dos tratamentos aplicados, empregando o programa de análises estatísticas SISVAR (Ferreira, 2000).

\section{RESULTADOS E DISCUSSÃO}

Como esperado, a figura 2 mostra que, independentemente da aplicação de calcário, a adição de esterco bovino proporcionou incrementos nos teores de matéria orgânica dos solos, os quais apresentaram ajuste quadrático; por outro lado, as doses de calcário não afetaram de modo significativo os teores de MO dos solos.

Em geral, para os solos RQo, LVAd-1 e LVAd-2, os valores mais altos do $\mathrm{P}$ Mehlich-1 foram obtidos para doses entre 6 e $8 \%$ de esterco bovino e doses em torno de 1,4 vez a dose calculada de calcário, após as quais observou-se tendência de redução nos teores de P. Para o solo LVd, a aplicação de esterco bovino não influenciou de maneira nítida os valores de P Mehlich-1 e a elevação das doses de calcário proporcionou incrementos nos teores de $\mathrm{P}$ até o nível 0,22 de calagem e $2,90 \%$ de esterco bovino, a partir das quais ocorreram respostas negativas (Figura 2).

A elevação dos valores de P Mehlich-1, com o aumento das doses de esterco bovino, pode ser atribuída ao bloqueio dos sítios de adsorção de $\mathrm{P}$ no solo, onde os grupos funcionais carboxílicos e fenólicos da matéria orgânica se ligam às hidroxilas dos óxidos de $\mathrm{Fe}$ e $\mathrm{Al}$ e complexam o $\mathrm{Al}$ em solução (Hue, 1991). Segundo Haynes (1984), o solo pode adsorver ácidos orgânicos com grande energia, competindo com sítios de adsorção de $\mathrm{P}$ e aumentando a disponibilidade desse nutriente para as plantas. A matéria orgânica pode originar, também, ligantes orgânicos liberados durante o processo de mineralização, que podem formar complexos com o Al ou complexos solúveis com o P da solução do solo, evitando que este seja adsorvido (Iyamuremye et al., 1996). Todavia, com as doses maiores de esterco bovino, verificou-se tendência de redução nos valores de $\mathrm{P}$ Mehlich-1. Isso se deveu, provavelmente, à adsorção de $\mathrm{P}$ aos compostos húmicos, dado o caráter aniônico da matéria orgânica, que, por via de pontes de cátions ( $\mathrm{Al}, \mathrm{Fe}$ e $\mathrm{Ca}$ ), podem reter o $\mathrm{P}$ (Novais \& Smyth, 1999).

$\mathrm{O} \mathrm{Al}$ e, ou, $\mathrm{Fe}$ em solução podem causar precipitação de fosfatos adicionados ao solo. A calagem, elevando o $\mathrm{pH}$ do solo pela produção de hidroxilas, promove a precipitação de $\mathrm{Fe}$ e de $\mathrm{Al}$ trocáveis, reduzindo a precipitação de $\mathrm{P}$ com esses dois metais. Entretanto, a retenção de $\mathrm{P}$ pelo solo mantém-se em valores estequiométricos semelhantes quando se substitui a precipitação do $\mathrm{P}$ pelo $\mathrm{Al}^{3+}$ do solo ácido pela sua adsorção pelo hidróxido de $\mathrm{Al}$ recém-formado pela calagem (Haynes, 1984). A elevação do $\mathrm{pH}$ promove, também, a desprotonação de hidroxilas de radicais orgânicos e daquelas expostas na superfície das argilas, aumentando a repulsão (menor adsorção) entre o fosfato e a superfície adsorvente e diminuindo o potencial eletrostático (Novais \& Smyth, 1999). Por outro lado, com o aumento do $\mathrm{pH}$, ocorre diminuição da forma $\mathrm{H}_{2} \mathrm{PO}_{4}^{-}$, em relação a $\mathrm{HPO}_{4}^{2-}$, esta (bivalente) preferencialmente adsorvida, contrabalançando o decréscimo do potencial eletrostático (Novais \& Smyth, 1999). Também, segundo esses autores, com elevada dose de calcário e maior teor de Ca trocável, pode ocorrer a precipitação de $\mathrm{P}$, formando fosfatos bi e tricálcicos de baixa solubilidade, explicando a redução nos valores de P Mehlich-1 com as doses mais elevadas de calcário.

A calagem de solos ricos em $\mathrm{Al}^{3+}$ tende a aumentar a adsorção de $\mathrm{P}$, diminuindo-a em solos pobres em $\mathrm{Al}^{3+}$, pois polímeros de hidróxidos de $\mathrm{Al}$ recém-formados com a calagem apresentam alta afinidade pelo $\mathrm{P}$ (Haynes, 1984). De acordo com esse autor, os polímeros catiônicos de $\mathrm{Al}$ formados com a calagem podem, também, capear colóides do solo, restringindolhes a carga negativa. De acordo com Novais \& Smyth (1999), considerando que, dentre as formas minerais ( $\mathrm{P}-\mathrm{Al}, \mathrm{P}-\mathrm{Fe}$ e $\mathrm{P}-\mathrm{Ca}$ ), o $\mathrm{P}-\mathrm{Al}$ é a que melhor representa o P lábil do solo, essa retenção não deve ser a pior opção quanto à disponibilidade de $\mathrm{P}$ às plantas.

Embora os solos não tenham sido comparados estatisticamente, os valores absolutos de P Mehlich-1 obedeceram à seguinte ordem crescente: LVd < LVAd-2 $<$ LVAd-1 < RQo (Figura 2). Isso pode ser explicado 


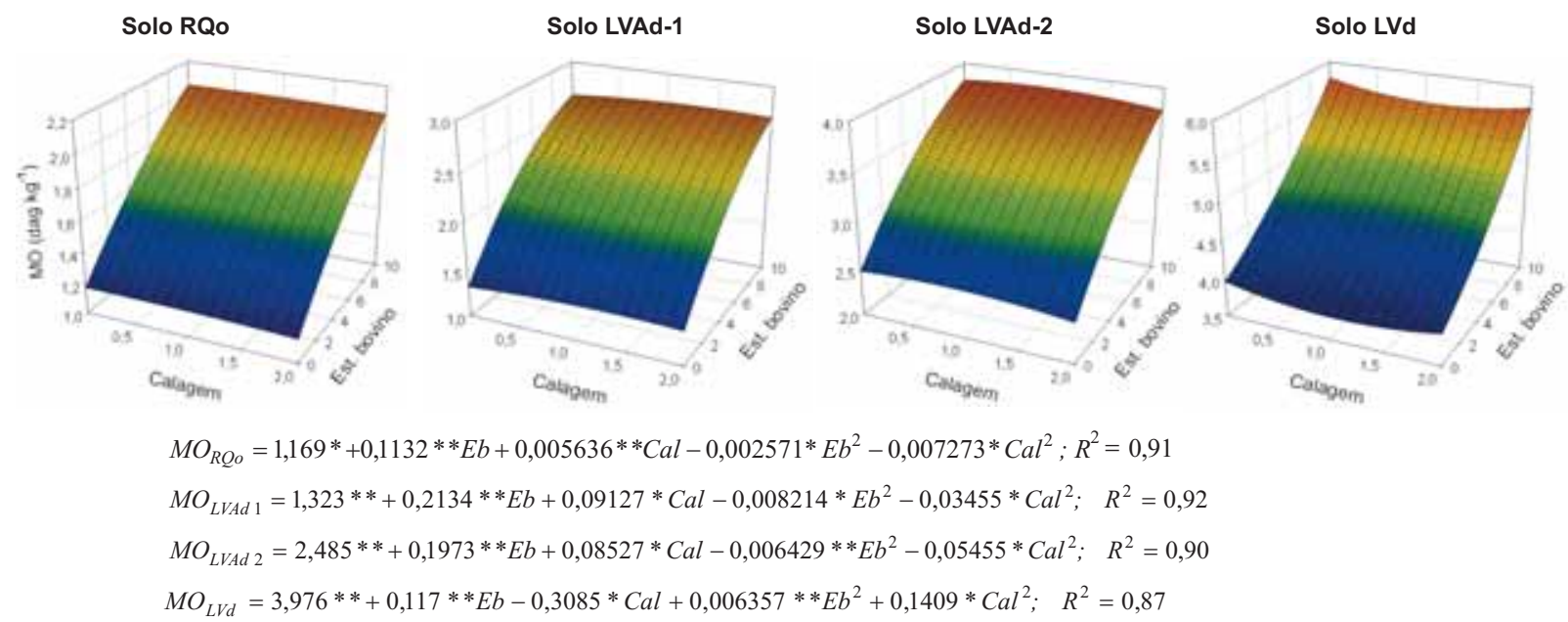

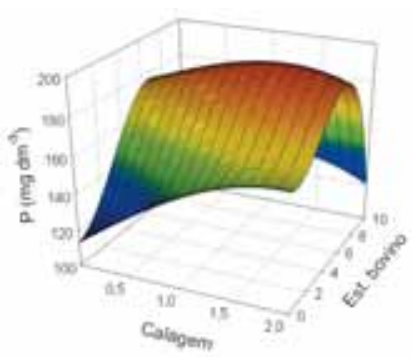

$$
\mathrm{P}_{\text {Máx }}=196,58 \mathrm{mg} \mathrm{dm}^{-3}
$$

$(E b=6,28 \% ; C a l=1,44 x)$

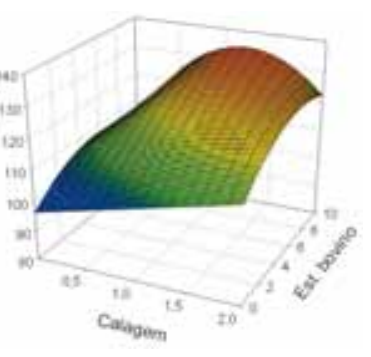

$\mathrm{P}_{\text {Máx }}=134,78 \mathrm{mg} \mathrm{dm}^{-3}$

$(E b=8,37 \% ; C a l=1,38 x)$

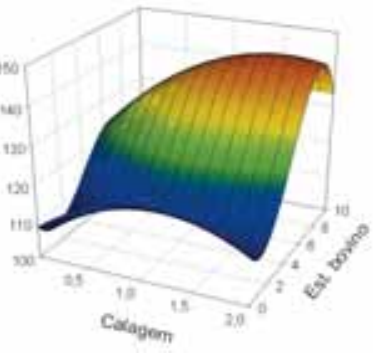

$\mathrm{P}_{\text {Máx }}=196,58 \mathrm{mg} \mathrm{dm}^{-3}$

$(E b=7,62 \% ; C a l=1,59 x)$

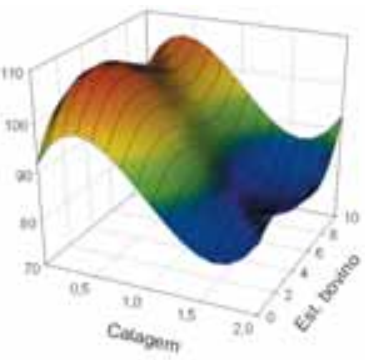

$\mathrm{P}_{\text {Máx }}=196,58 \mathrm{mg} \mathrm{dm}^{-3}$

$(E b=2,90 \% ; C a l=0,22 x)$

$P_{R Q o}=113,16 * *+2,04 n s E b+38,95 * * C a l+3,52 * * E b^{2}-0,36 * * E b^{3}-3,53 * * C a l^{3}-2,72 * * E b C a l ; \quad R^{2}=0,81$

$P_{\text {LVAd } 1}=96,16 * *-3,74 * E b-0,3 * * E b^{2}+8,21 * C a l+0,43 * * E b^{2} C a l-0,21 * E b^{2} C a l^{2} ; \quad R^{2}=0,98$

$P_{\text {LVAd } 2}=108,5 * *-6,02 * E b+2,71 * * C a l-0,22 * E b^{2}+20,94 * * C a l^{2}-9,37 * * E b^{2} C a l+0,15 * E b^{3} ; \quad R^{2}=0,72$

$P_{L V d}=91,74 * *+7,54 * E b-1,59 * * E b^{2}+0,09 * E b^{3}+40,23 * C a l-63,67 * * C a l^{2}+$

$20,92 * \mathrm{Cal}^{3}-2,21 * \mathrm{EbCal}+0,23 * E b^{2} \mathrm{Cal} ; \quad R^{2}=0,67$

Figura 2. Teores de matéria orgânica (MO) e P Mehlich-1 em função da aplicação de esterco bovino (Eb - \% do volume de solo) e calagem ( $\mathrm{Cal}$ - vezes a dose para elevar V = 60 \%) nos solos RQo, LVAd-1, LVAd-2 e LVd. **; * e ns: significativo a 1 e $5 \%$ e não-significativo, respectivamente.

pela maior capacidade de adsorção de $\mathrm{P}$ dos solos mais argilosos, que apresentam a seguinte ordem decrescente: LVd > LVAd-2 > LVAd-1 > RQo (Souza et al., 2006). Nesta ordem, os solos apresentam teores decrescentes de $\mathrm{MO}, \mathrm{Fe}_{\mathrm{d}}, \mathrm{Fe}_{\mathrm{o}}$, gibbsita e argila e crescentes de Ki e Kr (Quadro 1). À medida que os solos apresentam maior teor de óxidos, aumenta a adsorção de $\mathrm{P}$ e seu teor nas formas ligadas a $\mathrm{Al}$ e Fe. Os solos que apresentaram maiores valores de P Mehlich-1 corresponderam àqueles com maiores valores de $\mathrm{Ki}$ $\left(\mathrm{SiO}_{2} / \mathrm{Al}_{2} \mathrm{O}_{3}\right)$ e $\mathrm{Kr}\left(\mathrm{SiO}_{2} / \mathrm{Al}_{2} \mathrm{O}_{3}+\mathrm{Fe}_{2} \mathrm{O}_{3}\right)$ (Quadro 1).

A calagem e adubação orgânica, bem como a interação entre os dois fatores, influenciaram significativamente as formas de $\mathrm{P}$ em todos os solos estudados (Figura 3). Verificou-se que, de maneira geral, todas as formas de $\mathrm{P}$ mostraram comportamento crescente com o aumento nas doses de esterco bovino, independentemente das doses de calcário. Esses resultados corroboram os dados de Tiessen et al. (1992), os quais demonstram que a calagem e a adição de matéria orgânica alteram a dinâmica e as transformações do P no solo submetido a diferentes sistemas de manejo. Em estudos realizados em cafeeiros cultivados em Latossolo Roxo distrófico, foi observado que as principais transformações de $\mathrm{P}$ foram devidas às alterações nos valores de $\mathrm{pH}$ e nos teores de matéria orgânica do solo (Pavan \& Chaves, 1996). Resultados semelhantes foram obtidos por Zheng et al. (2004), estudando o efeito da aplicação de fertilizantes minerais e chorume sobre a dinâmica e as formas de $\mathrm{P}$ no solo. Vários outros trabalhos comprovam a influência da matéria orgânica sobre as transformações de P no solo (Hedley et al., 1982; Iyamuremye et al., 1996; Tokura et al., 2002). 

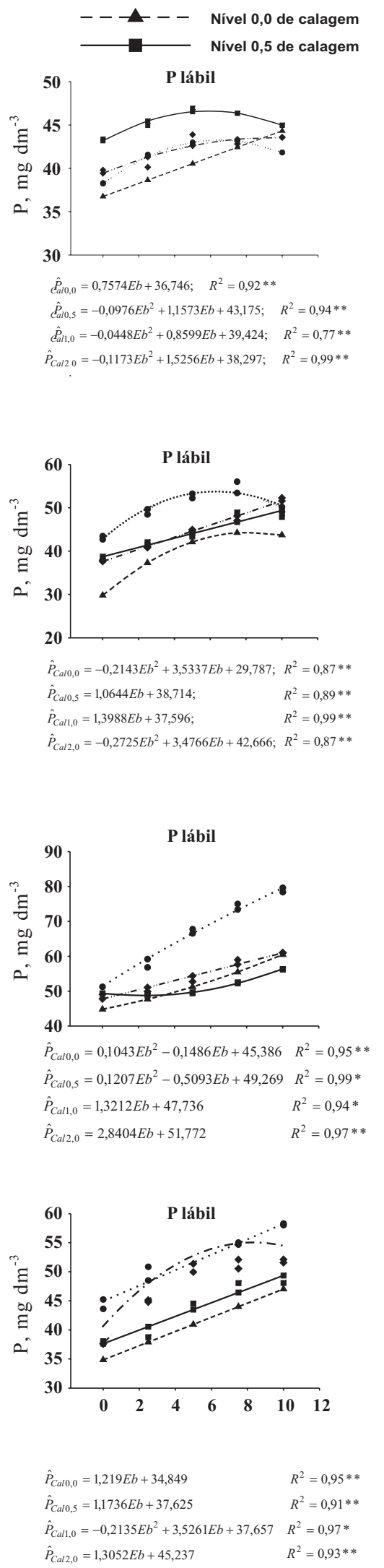

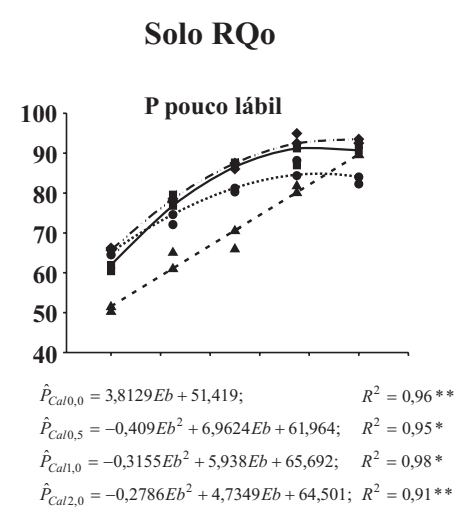

Solo LVAd-1

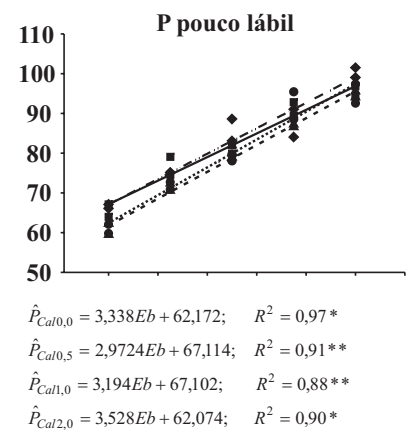

\section{Solo LVAd-2}

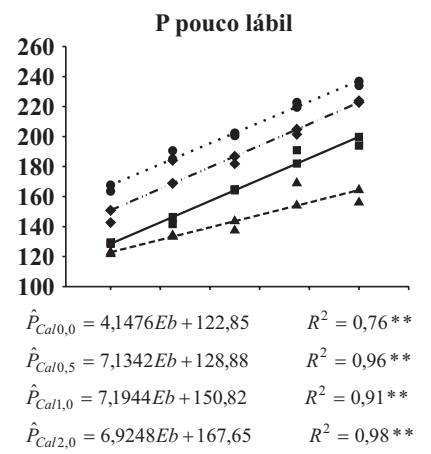

\section{Solo LVd}
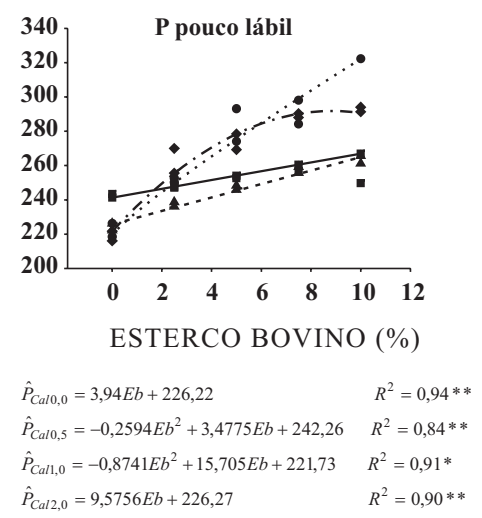
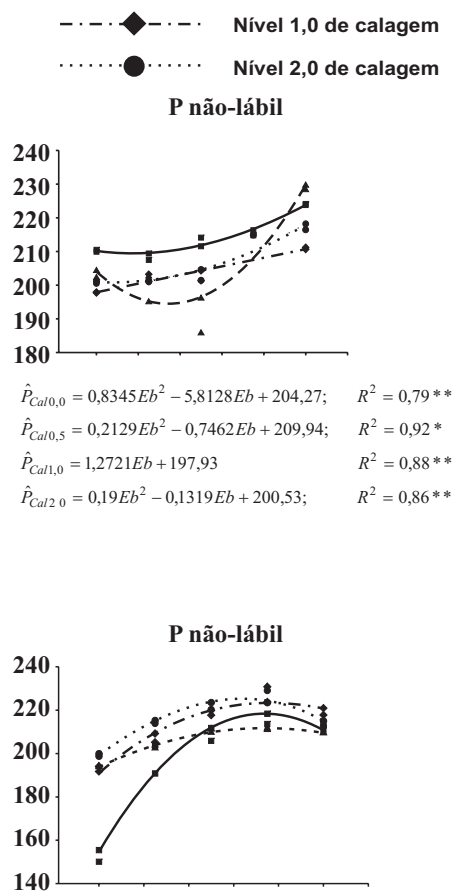

$\hat{P}_{C d 0,0}=-0,3255 E b^{2}+4,8168 E b+193,79 ; \quad R^{2}=0,98 *$ $\hat{P}_{\text {Cal } 0,5}=-1,1487 E b^{2}+17,008 E b+155,41 ; \quad R^{2}=0,91 * *$ $\hat{P}_{\text {Cal1 } 10}=-0,5444 E b^{2}+8,3651 E b+191,71 ; \quad R^{2}=0,89 * *$ $\hat{P}_{C a l 2,0}=-0,6649 E b^{2}+8,2794 E b+198,69 ; \quad R^{2}=0,89 * *$
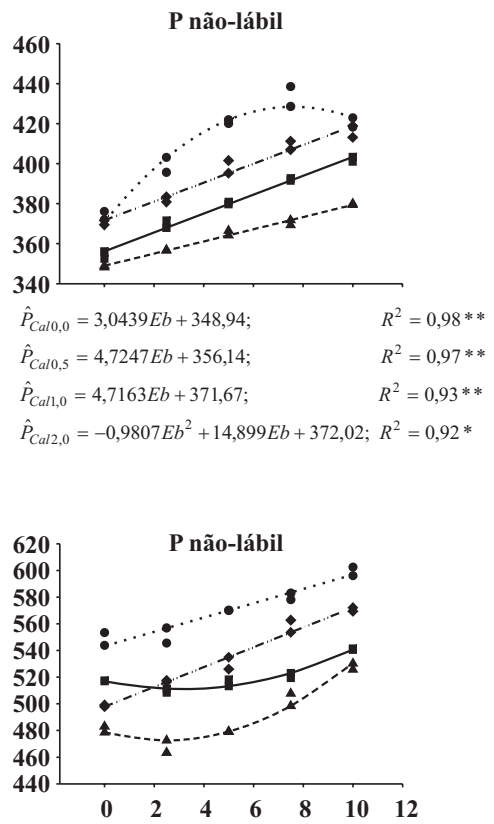

Figura 4. Distribuição das formas de $P\left(\mathrm{mg} \mathrm{dm}^{-3}\right)$ em amostras dos solos RQo, LVAd-1, LVAd-2 e LVd, em função da aplicação de esterco bovino $(E b \%)$, em cada nível de calagem $(C a l)$ **; *: significativo a 1 e $5 \%$, respectivamente. 
Quanto à contribuição das formas lábil, pouco lábil e não-lábil, independentemente dos tratamentos, verificou-se pequena participação da fração de $\mathrm{P}$ lábil no pool total de $\mathrm{P}$, predominando nesses solos as formas de P não-lábeis. Fernandes et al. (2002), trabalhando com solos de várzea do sul de Minas Gerais, e Ivanoff et al. (1998), com Histosols de Everglades, do sul da Flórida (EUA), também verificaram pequena participação do $\mathrm{P}$ lábil no $\mathrm{P}$ total dos solos. Segundo Walker \& Syers (1976), em solos altamente intemperizados, predominantes em regiões tropicais, a maior parte do $\mathrm{P}$ se encontra sob as formas nãolábil, oclusa ou, ainda, sob formas orgânicas estáveis. As formas não-lábeis de $\mathrm{P}$, compostas por frações relativamente insolúveis ligadas ao Ca e estáveis de Po e insolúveis de $\mathrm{Pi}$ e, portanto, não disponíveis em curto prazo, foram as responsáveis pela maior parte do $\mathrm{P}$ total nos solos.

As formas pouco lábeis de $\mathrm{P}$ ( $\mathrm{Pi}$ e $\mathrm{Po}-\mathrm{NaOH})$, com disponibilidade restrita à absorção vegetal, foram as que apresentaram o maior crescimento relativo, sendo essa fração uma importante fonte de $\mathrm{P}$ para a absorção vegetal. O hidróxido de sódio extrai formas de $\mathrm{P}$ consideradas de baixa disponibilidade para as plantas, associadas a fosfatos amorfos e cristalinos de $\mathrm{Fe} \mathrm{e} \mathrm{Al}$ (William et al., 1980); contudo, em solos de regiões tropicais, essas formas são relativamente dinâmicas (Tiessen et al., 1992), corroborando os dados de Araújo \& Salcedo (1997). Convém salientar que, em estudo de Araújo et al. (1993), realizado na zona canavieira do Nordeste, constatou-se que o $\mathrm{Pi}$ e $\mathrm{Po}-\mathrm{NaOH}$ contribuem bastante para o crescimento vegetal, indicando caráter lábil dessa forma de $\mathrm{P}$.

O aumento observado na fração de $\mathrm{Po}-\mathrm{NaOH}$ indica efeito de grande importância da aplicação de resíduo orgânico, pois esse reservatório representa um compartimento importante na ciclagem de $\mathrm{P}$ no solo (Tate \& Salcedo, 1988). Por processos de mineralização/imobilização o $\mathrm{P}$ orgânico pode se comportar como fator quantidade, tamponando as formas mais lábeis de $\mathrm{P}$ por meio do controle da mineralização (Tiessen et al., 1984). Essas formas são consideradas frações com disponibilidade restrita e se comportam como um reservatório (fator quantidade), representando um compartimento apreciável do nutriente no solo, sendo considerada como fonte e dreno (Hedley et al., 1982; Tiessen et al., 1984; Novais \& Smyth, 1999). Como a maior parte do $\mathrm{P}$ disponível às plantas é proveniente da matéria orgânica, os processos biológicos influenciam bastante a distribuição das formas de P no solo (Walbridge, 1991). Os processos biológicos regulam a dinâmica e distribuição de formas lábeis de $\mathrm{P}$, e a reciclagem da forma orgânica é um importante fator na disponibilização desse nutriente às plantas (Adepetu \& Corey, 1976).

Independentemente dos tratamentos, os valores de $\mathrm{P}$ lábil total ( $\mathrm{P}$ resina $+\mathrm{Pi}-\mathrm{NaHCO}_{3}+\mathrm{Po}-\mathrm{NaHCO}_{3}$ ) decresceram na seguinte ordem: LVAd-2 $>$ LVd $>$
LVAd-1 > RQo. Essa seqüência é inversa à observada para o P Mehlich-1, indicando que, dependendo do solo, esse extrator pode não extrair todas as formas lábeis de $\mathrm{P}$. Para os valores de $\mathrm{P}$ pouco lábil $(\mathrm{Pi}+\mathrm{Po}-\mathrm{NaOH}$ 0,1 e $0,5 \mathrm{~mol} \mathrm{~L}^{-1}$ ) e não-lábil (P-HCl + P-residual) a ordem decrescente foi: LVd > LVAd-2 > LVAd$1>$ RQo.

$\mathrm{O}$ aumento das formas de $\mathrm{P}$ pouco lábil com a aplicação de calcário pode ser devido à oxidação da matéria orgânica e aumento dos valores de Al e Fe amorfos nesses solos durante o período de incubação (Fernandes et al., 2002). Ainda, segundo Novais \& Smyth (1999), com a aplicação de calcário ocorre dispersão dos vários compostos presentes no solo pela elevação do $\mathrm{pH}$, expondo cargas elétricas positivas que adsorvem $\mathrm{P}, \mathrm{e}$, também, a formação de precipitados de $\mathrm{Al}$, que podem adsorver o $\mathrm{P}$. Os polímeros de hidróxidos de $\mathrm{Al}$ recém-formados apresentam alta afinidade por $\mathrm{P}$ (Haynes, 1984). A forma de P-HCl, que compreende as formas de $\mathrm{P}$ ligadas ao cálcio, embora considerada de baixa solubilidade em solos tropicais, não apresenta estabilidade irreversível, podendo ficar disponível à absorção vegetal em médio prazo (Araújo et al., 1993).

Nos quatro solos estudados, $\mathrm{o} P$ residual foi a forma que mais contribuiu para o pool de $\mathrm{P}$ não-lábil ( $\mathrm{P}$ $\mathrm{HCl}+\mathrm{P}$-residual), independentemente da aplicação de resíduo orgânico e, ou, calcário. Segundo Fernandes et al. (2002), Araújo et al. (1993) e Pavan \& Chaves (1996), esste resultado é esperado em solos ácidos. O $\mathrm{P}$ residual representa o P ocluso, retido pela hematita, goethita e gibbsita, o que explica os maiores teores dessa forma de $P$ nos solos mais intemperizados e com teores mais elevados de óxidos de Al e Fe. Para as formas de $\mathrm{P}$ lábil, pouco lábil e não-lábil houve influência da textura, mineralogia e do teor de matéria orgânica do solo nessas frações de $\mathrm{P}$, indicando que, para $o$ adequado manejo da adubação fosfatada, devemse considerar os atributos do solo ligados ao fator capacidade, independentemente da aplicação de resíduos orgânicos e, ou, calcário.

\section{CONCLUSÕES}

1. A adição de esterco bovino e de calcário incrementou os valores de P Mehlich-1, sendo essas alterações condicionadas à mineralogia e textura. Nos solos mais argilosos, intemperizados e com maior teores de óxidos, menores são os valores de $\mathrm{P}$ disponível.

2. Em geral, a adição de esterco bovino promoveu incrementos em todas as formas de $\mathrm{P}$ no solo.

3. Independentemente da aplicação de esterco bovino, as formas de $\mathrm{P}$ pouco lábeis (Po ligado a compostos húmicos e $\mathrm{Pi}$ ligado a $\mathrm{Fe}$ e $\mathrm{Al}$ ) foram as que apresentaram a maior contribuição para o $\mathrm{P}$ disponível às plantas. 
4. Predominaram as formas de P não-lábeis, sendo as alterações nos solos decrescentes na seguinte ordem de intensidade: LVd > LVAd-2 > LVAd-1 > RQo.

\section{LITERATURA CITADA}

ADEPETU, J.A. \& COREY, R.B. Organic phosphorus as a predictor of plant available phosphorus in soils of Southern Nigeria. Soil Sci., 122:159-164, 1976.

ALVAREZ V., V.H.; NOVAIS, R.F.; DIAS, L.E. \& OLIVEIRA, J.A. Determinação e uso do fósforo remanescente. B. Inf. SBCS, 25:27-32, 2000.

ALVAREZ V., V.H. Avaliação da fertilidade do solo (Superfícies de resposta - Modelos aproximativos para expressar a relação fator-resposta). Viçosa, MG, Universidade Federal de Viçosa, 1994. 75p.

ALVAREZ V., V.H. \& FONSECA, D.M. Definição de doses de fósforo para determinação da capacidade máxima de adsorção de fosfatos e para ensaios de casa de vegetação. R. Bras. Ci. Solo, 14:48-55, 1990.

ARAÚJO, M.S.B. \& SALCEDO, I.H. Formas preferenciais de acumulação de fósforo em solos cultivados com cana-deaçúcar na região Nordeste. R. Bras. Ci. Solo, 21:643-650, 1997.

ARAÚJO, M.S.B.; SALCEDO, I.H. \& SAMPAIO, E.V.S.B. Efeito de fertilizações fosfatadas anuais em solo cultivado com cana-de-açúcar. II. Formas disponíveis e efeito residual do P acumulado. R. Bras. Ci. Solo, 17:397-403, 1993.

DAY, P.R. Particle fractionation and particle-size analysis. In: BLACK, C.A., ed. Methods of soil analysis. Madison, America Society of Agronomy, 1965. v.1. p.545-566.

EMPRESA BRASILEIRA DE PESQUISA AGROPECUÁRIA EMBRAPA. Centro Nacional de Pesquisa de Solos. Sistema brasileiro de classificação de solos. Rio de Janeiro, 1999. $412 \mathrm{p}$.

EMPRESA BRASILEIRA DE PESQUISA AGROPECUÁRIA EMBRAPA. Serviço Nacional de Levantamento e Conservação de Solo. Manual de métodos de análise de solo. Rio de Janeiro, Ministério da Agricultura, 1997. 212p.

FERNANDES , L.A.; FAQUIN, V.; FURTINI, A.E. \& CURI, N. Formas de fósforo em solos de várzea e biodisponibilidade para o feijoeiro. Pesq. Agropec. Bras., 37:373-383, 2002.

FERREIRA, D.F. Análises estatísticas por meio do SISVAR (Sistema para Análise de Variância) para Windows versão 4. 0. In: REUNIÃO ANUAL DA REGIÃO BRASILEIRA DA SOCIEDADE INTERNACIONAL DE BIOMETRIA, 45., São Carlos, 2000. Anais. São Carlos, Universidade Federal de Carlos, 2000. p.255-258.

HAYNES, R.J. Lime and phosphate in the soil-plant system. Adv. Agron., 37:249-315, 1984.

HEDLEY, M.J.; STEWART, J.W.B. \& GHAUHAN, B.S. Changes in inorganic soil phosphorus fractions induced by cultivation practices and laboratory incubations. Soil Sci. Soc. Am. J., 46:970-976, 1982.
HUE, N.V. Effects of organic acids/anions on sorption and phytovailability in soils with different mineralogies. Soil Sci., 152:463-471, 1991.

IVANOFF, D.B.; REDDY, K.R. \& ROBINSON, S. Chemical fractionation of organic phosphorus in selected histosols. Soil Sci., 163:36-45, 1998.

IYAMUREMYE, F.; DICK, R.P. \& BAHAN, J. Organic amendments and phosphorus dynamics: II. Distribution of soil phosphorus fractions. Soil Sci., 161:436-443, 1996.

KHURI, A.I. \& CORNELL, J.A. Response surfaces: Designs and analyses. 2.ed. New York, Marcel Dekker, 1996. 510p.

KLUG, H.P. \& ALEXANDER, L.E. X-ray diffraction procedures for polycrystalline and amorphous materials. New York, John Wiley, 1974. 716p.

KRISHNA MURTI, G.S.R.; VOLK, V.V. \& JACKSON, M.L. Calorimetric determination of iron of mixed valency by orthophenantroline. Soil Sci. Soc. Am. Proc., 30:663-664, 1966.

MEHRA, O.P. \& JACKSON, N.L. Iron oxide removal from soils and clays by a dithionite-citrate system buffered with sodium bicarbonate. Clays Clays Miner., 3:317-327, 1960.

MURPHY, J. \& RILEY, J.P. A modified single solution methods for the determination of phosphae in natural waters. Anal. Chim. Acta, 26:31-36, 1962.

NOVAIS, R.F.\& SMYTH, T.J. Fósforo em solo e planta em condições tropicais. Viçosa, MG, Universidade Federal de Viçosa, 1999. 399p.

OLSEN, S.R. \& WATANABE, F.S. A method to determine a phosphorus adsorption maximum of soils as measured by the Langmuir Isotherm. Soil Sci. Soc. Am. Proc., 21:144149, 1957.

PAVAN, M.A. \& CHAVES, J.C.D. Alterações nas frações de fósforo no solo associadas com a densidade populacional de cafeeiros. R. Bras. Ci. Solo, 20:251-256, 1996.

R DEVELOPMENT CORE TEAM. R: Language and environment for statistical computing. $\mathrm{R}$ Foundation for Statistical computing, Vienna, Austria. 2004. Disponível em: <http://www.R-project.org>. acesso em: 25 dez. 2004.

RAIJ, B. van.; QUAGGIO, J.A.; CANTARELLA, H.; FERREIRA, M.E.; LOPES, A.S. \& BATAGLIA, O.C. Análise química do solo para fins de fertilidade. Campinas, Fundação Cargil, 1987. 170p.

SCHWERTMANN, U. Differenzierung der eisenoxide des bondes durch extraktion mit ammonium-oxalat-losung. Zeitschrift Pflanz., 105:194-202, 1964.

SILVA, F.C. \& RAIJ, B. van. Disponibilidade de fósforo em solos avaliada por diferentes extratores. Pesq. Agropec. Bras., 34:267-288, 1999

SOUZA, R.F.; FAQUIN, V.; TORRES, P.R.F. \& BALIZA, D.P. Calagem e adubação orgânica: influência na adsorção de fósforo em solos. R. Bras. Ci. Solo, 30:975-983, 2006.

TATE, K.R. \& SALCEDO, I.H. Phosphorus control of soil organic matter accumulation and cycling. Biogeochem., 5:99-107, 1988. 
TIESSEN, H.; SALCEDO, I.H. \& SAMPAIO, E.V.S.B. Nutrients and soil organic matter dynamic under shiftig cultivation in semi-arid northeastern Brazil. Agric. Ecosyst. Environ., 39:139-159, 1992.

TIESSEN, H.; STEWART, J.W.B. \& COLE, C.V. Pathways of phosphorus transformations in soils of differing pedogenesis. Soil Sci. Soc. Am. J., 48:853-858, 1984.

TOKURA, A.M.; FURTINI NETO, A.E.; CURI, N.; FAQUIN, V.; KURIHARA, C.H. \& ALOVISI, A.A. Formas de fósforo sob plantio direto em razão da profundidade e tempo de cultivo. Pesq. Agropec. Bras., 37:1467-1476, 2002.

TURRIÓN, M.B.; GLASER, B. \& SOLOMON, D. Effects of deforestation on phosphorus pools in mountain soils of the Alay Range, Khyrgyzia. Biol. Fert. Soils, 31:134-142, 2000.
WALBRIDGE, M.R. Phosphorus availability in acidic organic soils of the lower North Carolina coastal plain. Ecology, 72:2083-2100, 1991.

WALKER, T.W. \& SYERS, J.K. The fate of phosphorus during pedogenesis. Geoderma, 15:1-19, 1976.

WILLIAM, J.D.H.; MAYER, T. \& NRIAGU, J.O. Extractability of phosphorus minerals common in soils and sediments. Soil Sci. Soc. Am. J., 44:462-465, 1980.

ZHENG, Z.; McLEOD, J.A.; SANDERSON, J.B. \& LAFOND, J. Soil phosphorus dynamics after annual applications of mineral fertilizers and liquid dairy manure: Fractionation and path analyses. Soil Sci., 169:449-456, 2004. 